\title{
TURBULENT BEHAVIOR OF FLUIDIZED SEDIMENTS IN COMPOSITE SHEAR FLOW
}

\author{
Ayumi Saruwatari ${ }^{1}$, Wataru Matsuzaki ${ }^{2}$ and Yasunori Watanabe ${ }^{3}$
}

\begin{abstract}
A particle imaging measurement of granular particles was applied to fluidized and suspended solid particles involved in steady and unsteady shear flows. In this measurement, $42 \%$ sodium iodide solution was used as a fluid medium to coincide the refraction index with the transparent bed material (silica gel). Therefore, the vertical distributions of the granular velocity and turbulent behavior within the bed can be measured by tracking the dyed particles mixed with the bed material. The turbulent kinetic energy in the fluidized layer and particle concentration can also be measured using this technique. The turbulence developed over the bed disturbed the bed material, and as a result the surface particles were lifted and suspended. The underlying mechanism of fluidization and suspension of the sediment seabed in complex turbulent shear flow is believed to be understood through further parametric studies based on the present imaging technique.
\end{abstract}

Keywords: granular bottom particles; suspension and fluidization; refractive index matching technique

\section{INTRODUCTION}

It is well known that shear flow reduces contact stress between granular bottom particles to fluidize and suspend the particles, which enhances the sediment transport. The transportation of the sediment is described by the following equation.

$$
\frac{\partial \bar{C}}{\partial t}+\frac{\partial}{\partial x_{i}} \bar{C} \overline{u_{p i}}+\frac{\partial}{\partial x_{i}} \overline{C^{\prime} u_{p i}^{\prime}}=S
$$

Here, $C$ is the sediment concentration, $u_{p i}$ is the velocity tensor of the bottom particles, and overline and prime represent the mean and fluctuation components, respectively. $S$ denotes the pickup rate of the sediment under turbulent shear flow, which is well parameterized by Shields number (Nielsen, 1992). The third term in the left-hand side represents the turbulent diffusion of the sediment concentration. This term is generally modelled as follows.

$$
\frac{\partial}{\partial x_{i}} \overline{C^{\prime} u_{p i}^{\prime}}=-\nabla(D \nabla \bar{C})
$$

If we can measure the particle velocity, we may be able to directly parameterize this term.

A lot of studies about the turbulence developed at the bottom have been experimentally performed (e.g. Lamb et al., 2004, Manes et al. 2009). Although flow properties near the granular bottom can be known by using ultrasonic anemometer, turbidity meter, pore pressure gauge and so on, direct measurement of granular bottom particles is difficult since the number density of the particles is too high near the bed.

In this study, we visually measure the velocity and turbulence of the granular bottom particles in turbulent shear flow. The fundamental features about the velocity and turbulence in the fluidized granular bottom are investigated.

\section{REFRACTIVE INDEX MATCHING}

We used a refractive index matching technique to visualize the flow of granular bottom particles moving in shear flows. As shown schematically in Fig. 1, when we take an image of a dyed particle within transparent particles, it enable us to know the precise location of the dyed particle since the optical axis is refracted at the interfaces of the transparent particles. When the refractive index of the particles coincides with that of the surrounding fluid, however, the optical axis does not refract at the interfaces of the particles. Then the granular particles become optically transparent, and we can directly know the location of the dyed particle from the captured image.

In this study, silica gel particles with a refractive index of 1.46 and diameter of $1.0 \mathrm{~mm}$ were used as a bed material, and $42 \%$ sodium iodide (NaI) solution was used for the liquid phase. The NaI solution, whose refractive index varies with the concentration, was made to have the same refraction

\footnotetext{
${ }^{1}$ Faculty of Engineering, Hokkaido University, N13 W8, Sapporo, 060-8628, Japan

2 Graduate School of Engineering, Hokkaido University, N13 W8, Sapporo, 060-8628, Japan

${ }^{3}$ Faculty of Engineering, Hokkaido University, N13 W8, Sapporo, 060-8628, Japan
} 


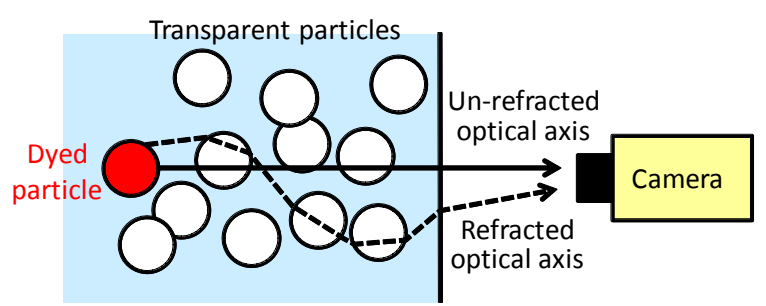

Figure 1. Schematic image of optical axes in a mixture of transparent particles and liquid.

\begin{tabular}{|c|c|c|c|}
\hline & Sediments & $\begin{array}{l}\text { Silica gel } \\
\text { particles }\end{array}$ & Grass beads \\
\hline Refractive index & - & 1.46 & $>1.5$ \\
\hline Specific gravity & $2.0-2.7$ & 2.4 & $2.5-2.7$ \\
\hline $\begin{array}{c}\text { Angle of } \\
\text { response [deg] }\end{array}$ & $20-60$ & 37.0 & $20-40$ \\
\hline
\end{tabular}

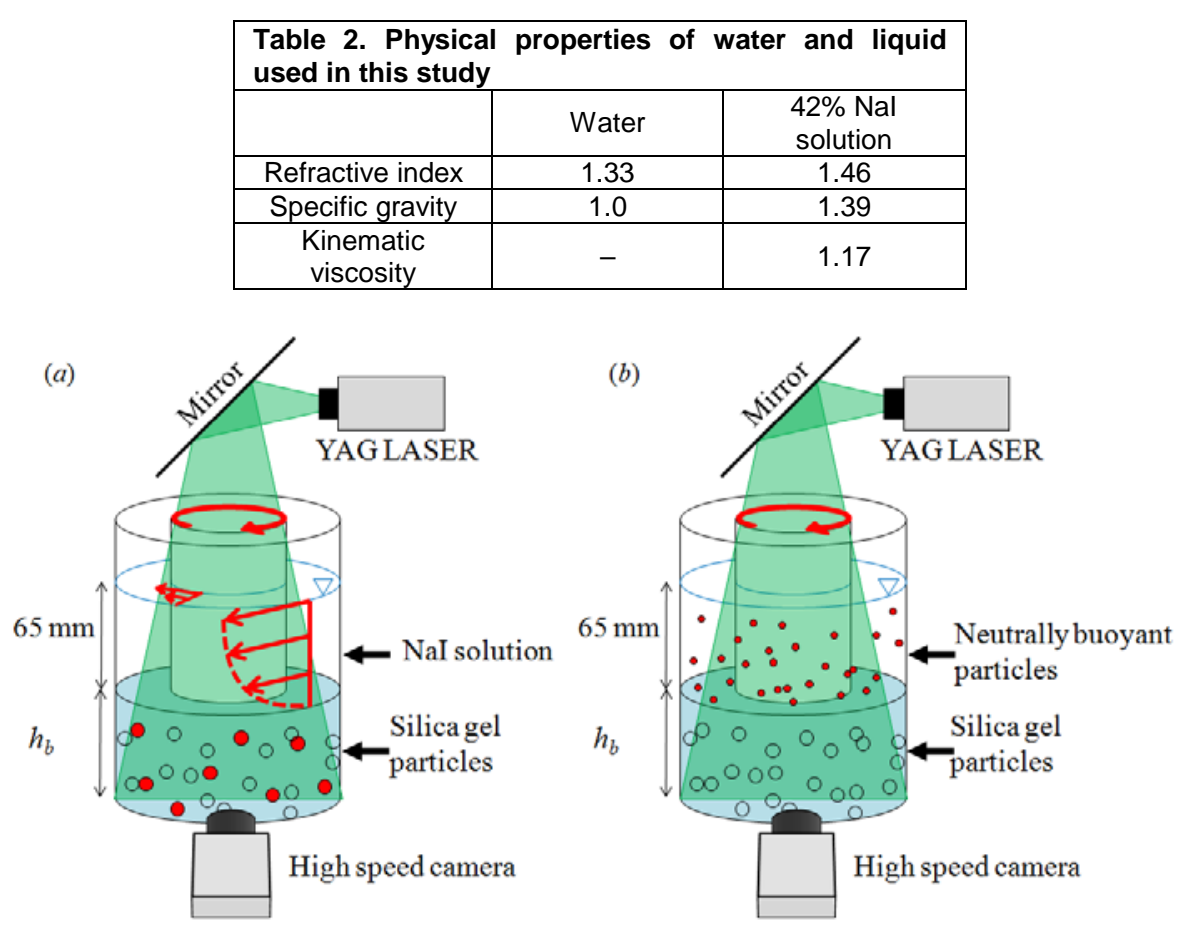

Figure 2. Experimental setup for measuring granular bottom particles (a) and liquid phase (b).

index as the silica gel particles. The physical properties of the NaI solution and silica gel particles are shown in Table 1 and Table 2. The specific gravity of the silica gel particles is roughly identical to the sediments, and the angle of response is in the range of that of the sediments.

\section{EXPERIMENTAL SETUP}

The granular bed materials (silica gel) were layered at 0, 15 and $30 \mathrm{~mm}$ thicknesses between two concentric cylinders with the radii of 45 and $75 \mathrm{~mm}$ (Fig. 2). The $42 \% \mathrm{NaI}$ solution was filled within the outer cylinder to be the depth of $65 \mathrm{~mm}$. Therefore, there was no refraction and reflection between the interfaces of the liquid and particles - the granular particles became completely invisible. The fluorescently dyed particles of silica gel, which were preliminarily mixed within the bed materials, were illuminated by the Laser light sheet of a YAG Laser. The location of the Laser sheet was fixed at 20 $\mathrm{mm}$ from the inner cylinder. The fluorescent light emitted from the dyed particles was recorded by a high-speed camera with the recording frequency of $250 \mathrm{~Hz}$, a shutter speed of 1/250 sec, an FOV of 84 $\mathrm{mm} \times 66 \mathrm{~mm}$ and a 1280 pixel x 1024 pixel resolution. Neutrally buoyant particles were also mixed 


\begin{tabular}{|c|c|c|c|}
\hline \multicolumn{4}{|c|}{ Table 3. Experimental conditions } \\
\hline Case \# & $\begin{array}{c}\text { Granular bed } \\
\text { thickness [mm] }\end{array}$ & $\begin{array}{c}\text { Rotation } \\
\text { frequency [rpm] }\end{array}$ & $\begin{array}{c}\text { Measurement } \\
\text { Object }\end{array}$ \\
\hline 1 & 0 & 150 & \\
2 & 0 & 200 & \\
3 & 15 & 150 & \\
4 & 15 & 200 & \\
5 & 15 & 300 & Liquid velocity \\
6 & 15 & 350 & \\
7 & 30 & 110 & \\
8 & 30 & 150 & \\
9 & 30 & 200 & \\
10 & 30 & 300 & \\
11 & 30 & 150 & Particle velocity \\
12 & 30 & 200 & \\
13 & 30 & $-200-+200$ & \\
\hline
\end{tabular}

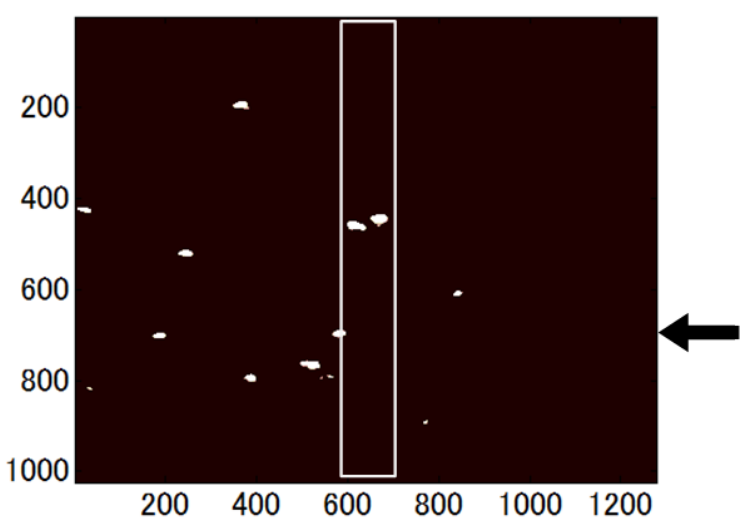

Figure 3. Typical image obtained in this experiment. Arrow indicates the initial bottom level and white line indicates the band-shaped region of $1 \mathrm{~cm}$ width where we measured the velocity.

within the liquid in order to measure the fluid flow. The vertical direction is represented by the $z$-axis with its origin at the initial granular bottom level.

The inner cylinder was rotated to generate Couette flow within the cylinder walls and also the bottom boundary shear flow. The experimental conditions with various rotation frequencies and initial granular bottom thicknesses are shown in Table 3. The typical image taken in this experiment is shown in Fig. 3. The standard particle tracking velocimetry (PTV) was used for the recorded particles to estimate the velocity and turbulence statistics. The velocity in the band-shaped region of $1 \mathrm{~cm}$ width was used to obtain the vertical profiles of the velocity.

\section{RESULTS}

\section{Velocity Profiles}

Figure 4 shows the vertical profiles of the concentration of the bottom particles, and the horizontal velocity of the particles and liquid. Flow field was successfully measured near the granular bottom, where the image measurement had been difficult due to the high concentration of the granular particles. Granular bottom was fluidized near the initial bottom level, and bottom particles were suspended in the region up to $z<42 \mathrm{~mm}$ in this experimental condition. The velocity of the particles and liquid were gradually changed near the bottom. We define this region using a similar idea with the "mixing layer" to characterize the transition of the velocity profiles. In the velocity distribution that changes from zero to the maximum velocity, we defined the mixing layer as the region where the mean velocity is $10-90 \%$ of the maximum velocity. We also defined this region as the fluidized layer.

The thickness of the mixing layer and mean shear rate in the mixing layer were compared with the rotation frequency of the cylinder in Fig. 5. An increase in the rotation frequency caused no significant change of the mean shear rate in the mixing layer, while the thickness of the mixing layer increased with 
the rotation frequency. The mixing layer thickness over the granular bottom was greater than that over the flat bottom. The mixing layer of the granular particle velocity became twice as thick as that of

(a) case $11 ; 150 \mathrm{rpm}$

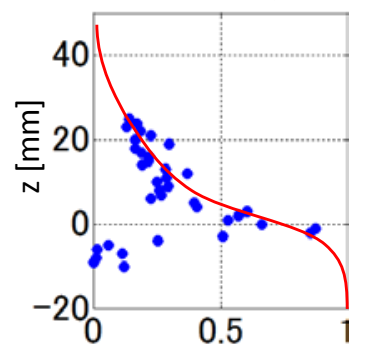

Particle concentration

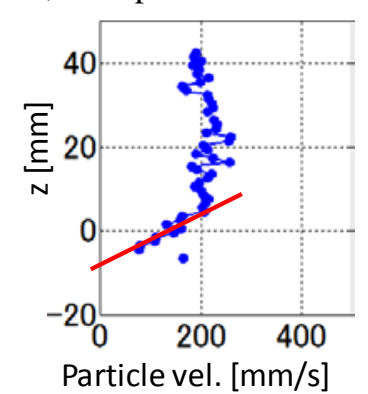

Particle vel. [mm/s]

(c) case 12; $200 \mathrm{rpm}$

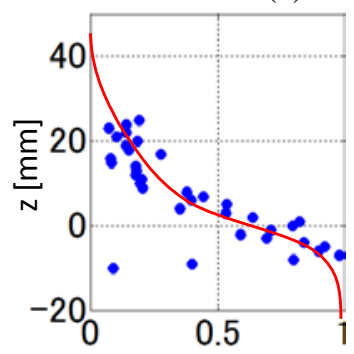

Particle concentration

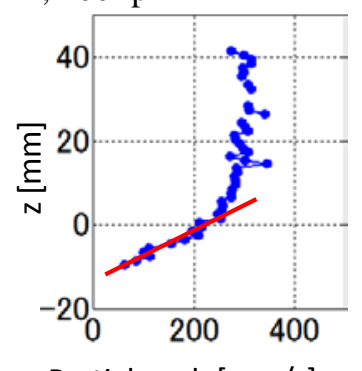

Particle vel. [mm/s] (b) case 8; $150 \mathrm{rpm}$

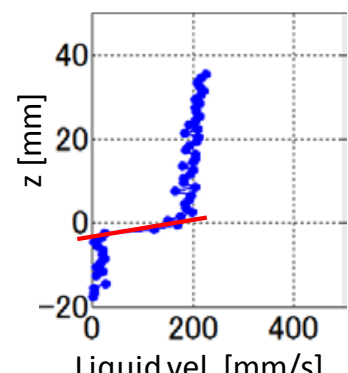

Liquid vel. $[\mathrm{mm} / \mathrm{s}]$

(d) case 9; 200 rpm

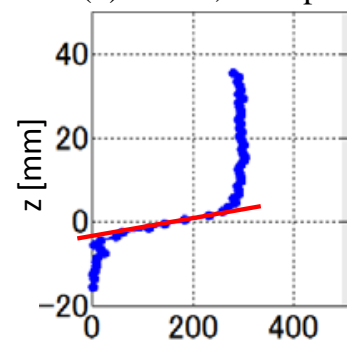

Liquid vel. [mm/s]

Figure 4. Vertical profiles of the number density and mean horizontal velocity of the granular particles (a), (c), and horizontal velocity of the liquid (b), (d).
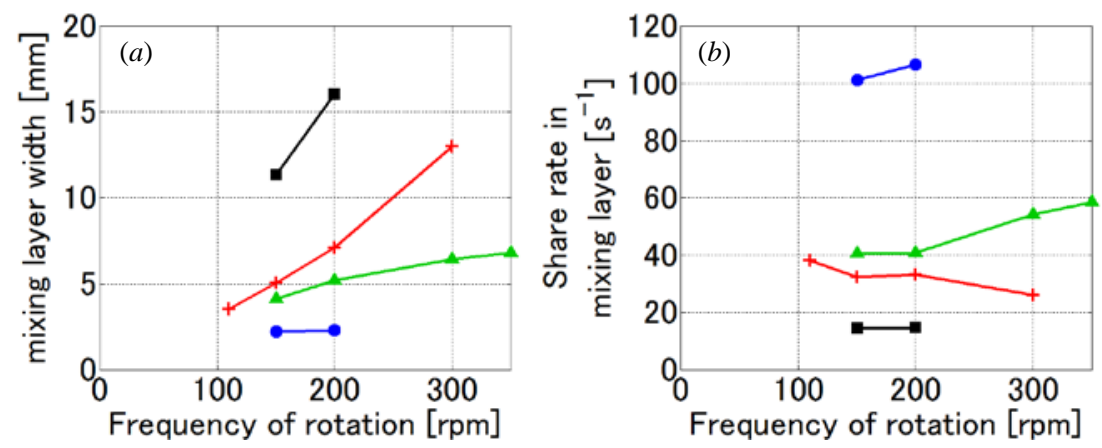

Figure 5. Relation between the thickness of the mixing layer and rotation frequency (a) and shear rate in the mixing layer and rotation frequency (b). $\bullet$ : Liquid velocity over the flat bottom, $\mathbf{\Delta}$ : Liquid velocity over the granular bed with $15 \mathrm{~mm}$ thickness, +: Liquid velocity over the granular bed with $30 \mathrm{~mm}$ thickness, a: Particle velocity of granular bed with $\mathbf{3 0} \mathrm{mm}$ thickness.

the liquid velocity field, since the velocity field of the bottom particles was more disturbed due to the collision between the particles.

Turbulent Kinetic Energy

We calculated the turbulent kinetic energy (TKE) of the liquid and particle velocities using the following equation.

$$
E=\frac{1}{2}\left(\overline{u^{\prime 2}}+\overline{w^{\prime 2}}\right)
$$

Here, $u^{\prime}=u-\bar{u}, w^{\prime}=w-\bar{w}$ are velocity fluctuation into the horizontal and vertical directions ( $u$ and $w$ are measured horizontal and vertical velocities, $\bar{u}$ and $\bar{w}$ are mean velocities). Figure 6 shows the 
vertical profiles of the TKE. The TKE over the flat bottom became high just near the bottom, while that over the granular bottom increased throughout the mixing layer. The turbulent diffusion near the

(a)

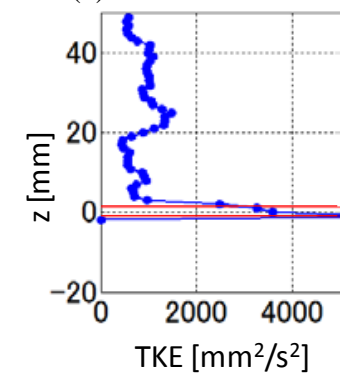

(b)

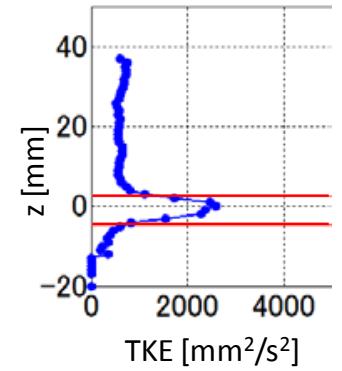

(c)

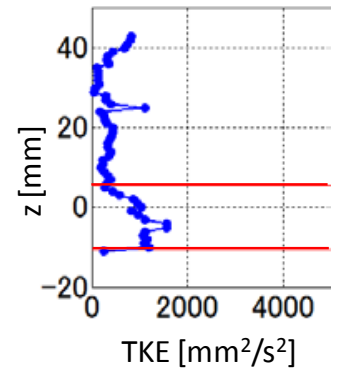

Figure 6. Vertical profiles of the turbulent kinetic energy. TKE of the liquid velocity over the flat bottom (a, case 2), liquid velocity over the granular bottom (b, case 9), and particle velocity of the granular bottom (c, case 12) Lines in the figures indicate the range of the mixing layer.
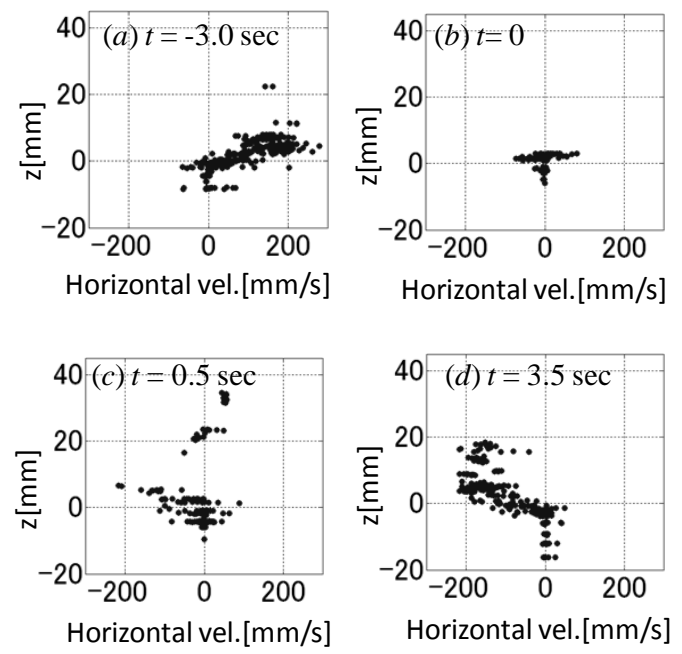

Figure 7. Evolution of the velocity profile of the bottom particles in unsteady shear flow (case 13).

granular bottom causes the suspension and fluidization of the bottom particles. Since the suspended and fluidized particles drag the surrounding liquid, the liquid velocity over the granular bottom is diffused and the mixing layer becomes thicker than the cases of the flat bottom.

\section{Unsteady Flow Over the Granular Bed}

We made a sudden change to the rotating direction of the cylinder as an example of an extreme unsteady flow, and measured the bottom particle velocity. Figure 7 shows the evolution of the vertical profiles of the horizontal velocity of the bottom particles before and after the sudden change of the flow direction. The time when the flow direction is changed is defined at $t=0$. The particles' concentration over the granular bed varied with time. The particles were lifted mostly upward at $t=0.5 \mathrm{sec}$ through this observation. Sediment transport in the sudden change of the flow field is important since most sediment diffusion occurs when vortices caused by breaking waves arrive at sea bed. Therefore, further investigation to determine the effects of the unsteady turbulence on sediment disturbances is needed to better understand sediment dynamics in the surf zone.

\section{CONCLUSION}

Refractive index matching technique was applied to the flow of the granular bottom particles in sheared flows. We confirmed that the velocity and turbulence of the fluidized and suspended particles can be measured directly using this technique. Further experiments should be done to investigate the turbulent velocity field developed near the granular bottom. 


\section{REFERENCES}

Lamb, M.P., E. D'Asaro and J.D. Parsons. 2004. Turbulent structure of high-density suspensions formed under waves. J. Geophys. Res., 109, C12026.

Manes, C., D. Pokrajac, I. McEwan and V. Nikora. 2009. Turbulence structure of open channel flows over permeable and impermeable beds: A comparative study. Phys. Fluids, 21, 125101.

Nielsen, P. 1992. Coastal bottom boundary layers and sediment transport. Advanced Series on Ocean Engineering, 4. 